\title{
Control-Matrix Approach to NCSX Design
}

\author{
H.E. Mynick, N. Pomphrey \\ Plasma Physics Laboratory, Princeton University \\ P.O. Box 451 \\ Princeton, New Jersey 08543-0451, U.S.A.
}

\begin{abstract}
The control matrix approach to stellarator design yields a description of the combinations of 'control knobs' $Z_{j}$ which can be adjusted to provide independent control of physics figures of merit $P_{i}$ (e.g., ripple levels or kink growth rates), as well as those combinations which affect none of these $P_{i}$. This can be used both in finding superior design points, as well as in using a configuration's control knobs to have good operational flexibility about those design points. We have set up the machinery needed to perform this analysis, and here present initial results of its application to some candidate Quasi-Axisymmetric (QA) configurations. In the process of the analysis, a first exploration of the topography of the configuration space $\mathbf{Z}$ in the vicinity of these candidate systems has been performed, whose character is discussed.
\end{abstract}




\section{Introduction}

In developing candidate configurations for an attractive QA stellarator (QAS)[1], the NCSX group has relied heavily on an automated optimizer, which conducts a search in a parameter space $\mathbf{Z}=\left\{Z_{j}\right\}\left(j=1, . . N_{z}\right)$ describing the stellarator boundary, using an objective function $F(P)$ which is a function of figures of merit $\mathbf{P}=\left\{P_{i}(\mathbf{Z})\right\}\left(i=1, . . M_{p}\right)$ characterizing the physics properties (e.g., transport, kink stability, etc.) of the configuration. While a powerful tool, the optimizer is searching a space whose topography has been essentially unknown, and we have rather limited understanding of why the optimizer arrives at the design points $\mathbf{Z}_{0}$ it does. Deeper insight into this would enhance the our ability to locate attractive design points, e.g., by reducing the dimensionality of the $\mathbf{Z}$-space, or by recognizing systematic ways in which the topography can lead the optimizer to local but inferior optima of $F$.

The control matrix project discussed here is intended to provide this insight, through increased understanding of the topography, and through applying mathematical techniques such as SVD methods to gain a clear grasp of how the $P_{i}(x)$ can be changed to achieve superior base configurations and operational flexibility.

\section{Formulation}

As required by the VMEC code we employ to compute our equilibria, for the studies discussed here we specify a single stellarator configuration $\mathbf{Z}$ by the set $\mathbf{Z}=\left(R_{m n_{1}}, Z_{m n_{1}}, R_{m n_{2}}, \ldots Z_{m n_{N / 2}}\right)$ of Fourier amplitudes which describe the plasma boundary $[R(\theta, \zeta), Z(\theta, \zeta)]$. For $\mathrm{C} 10$, the first base configuration of our study, one has $N \equiv N_{z}=2 \times 39=78$ amplitudes. Using the same codes as employed by the optimizer, we compute $M \equiv M_{p}=5$ figures of merit $\mathbf{P}=$ $\left(\chi_{1}^{2}, \chi_{2}^{2}, W_{1}, W_{2}, \lambda\right)$, where $P_{1-4}$ are 4 measures of the ripple strength, and hence the level of nonaxisymmetric transport one may expect, and $P_{5} \equiv \lambda=\omega^{2}$ is the kink eigenvalue from TERPSICHORE, negative for unstable modes. More specifically, $\chi_{1,2}^{2} \equiv \chi^{2}\left(s_{1,2}\right)$, with $\chi^{2}(s) \equiv \sum_{m, n \neq 0} B_{m n}^{2} / B_{00}^{2}, W_{1,2} \equiv W\left(s_{1,2}\right)$ is the 'water function' [2] measuring the average ripple-well depth, and $s_{1,2}=$ $1 / 2,1 / \sqrt{2}$, with $s \equiv \psi / \psi_{a}$ the toroidal flux, normalized to unity at the plasma edge. Other figures of merit might be usefully added to this set, such as ballooning growth rate, or surface quality, and the same formal machinery employed to study any such set.

Expanding $\mathbf{P}\left(\mathbf{Z}=\mathbf{Z}_{0}+\mathbf{z}\right)=\mathbf{P}\left(\mathbf{Z}_{0}\right)+\mathbf{p}$ about $\mathbf{Z}=\mathbf{Z}_{0}$, one has (writing in 
component-form, with summation over repeated indices assumed)

$$
p_{i}\left(\mathbf{Z}_{0}+\mathbf{z}\right)=G_{i j}\left(\mathbf{Z}_{0}\right) z_{j}+\frac{1}{2} H_{i j k}\left(\mathbf{Z}_{0}\right) z_{j} z_{k}+(\text { h.o. }),
$$

with h.o. $\equiv$ higher-order terms. For small enough z, one has the matrix equation

$$
\mathbf{p}=\mathbf{G}_{0} \cdot \mathbf{z}
$$

with $\mathbf{G}_{0} \equiv \mathbf{G}\left(\mathbf{Z}_{0}\right)$ the $M \times N$ 'control matrix' at design point $\mathbf{Z}_{0}$. This may be inverted, using the SVD theorem[3]

$$
\mathbf{G}_{M \times N}=\mathbf{U}_{M \times N} \cdot \mathbf{W}_{N \times N} \cdot \mathbf{V}_{N \times N}^{T},
$$

with $\mathbf{U}, \mathbf{V}$ unitary matrices, and $\mathbf{W}$ a diagonal matrix. This theorem permits one to invert the nonsquare matrix $\mathbf{G}$, and provides bases spanning the its range and nullspace.

Taking the particular basis set $\boldsymbol{\pi}^{i=1, M}$ in the target $\mathbf{P}$-space to be the set of unit vectors with 1 in the $i^{t h}$ position and 0 elsewhere, one has the corresponding set $\boldsymbol{\xi}^{i}$ of displacements in $\mathbf{Z}$-space

$$
\boldsymbol{\xi}^{i} \equiv \mathbf{G}_{0}^{-1} \cdot \boldsymbol{\pi}^{i},
$$

physically representing displacements which change a single physics parameter $P_{i}$ by unity, leaving the others unchanged. These span the range of $\mathbf{G}$. The $(N-M)$ vectors spanning the nullspace of $\mathbf{G}$ (change the configuration without modifying any of the $P_{i}$ ) are also important. Here, we shall focus mostly on the $\boldsymbol{\xi}^{i}$.

Figs. 1 show contour and surface plots of $\boldsymbol{\xi}^{1}$ and $\boldsymbol{\xi}^{5}$ over in the $(\theta, \tilde{\zeta})$-plane from this SVD analysis. (Here, $\tilde{\zeta} \equiv N_{f p} \zeta$.) For compactness, we show a plot of the single function $\xi^{i}(\theta, \tilde{\zeta}) \equiv \xi_{R}^{i}+\xi_{Z}^{i}$, the sum of the $R$ and $Z$ components of $\boldsymbol{\xi}^{i} \equiv \hat{R} \xi_{R}^{i}+\hat{Z} \xi_{Z}^{i}$. The contours of these separately are similar, but have even and odd symmetry, respectively, about $(\theta, \tilde{\zeta})=(0,0)$. Interestingly, though the eigenvalues $w_{i}$ of matrix $\mathbf{W}$ show these are not nearly collinear, the contours of the $\xi^{i}$ for the other 3 transport figures of merit resemble those of $\xi^{1}$, and these are rather different from that for the kink $\left(\xi^{5}\right)$, which is somewhat more elongated toroidally.

The effect of these on the unperturbed $\mathrm{C} 10$ boundary for $\tilde{\zeta}=0$ and $\pi$ is shown in Fig. 2. One notes in particular that $\xi^{5}$ for diminishing the kink produces an indentation of the outboard side at the half-period $\tilde{\zeta}=\pi$, enhancing the (negative) triangularity which that cross-section possesses. This is consistent with the earlier empirical observation[1] that kink stability can be helped by providing such an indentation. 


\section{Topography of Z-space}

The validity of Eqs. (1) or (2) depends on the typical scales of variation in $\mathbf{Z}$-space of the $P_{i}$, which up to now have been essentially unknown. We have assessed this variation for all $78 Z_{j}$ for the $P_{i}$ presently being used, in the vicinity of the C10-C82 family of configurations.

Some typical results are shown in Fig. 3, showing the variation of $P_{1}=\chi_{1}^{2}$ and $P_{5}=\lambda$ with deformation amplitude (in meters), for changes $z_{j} \equiv \delta Z_{j}$ of the given $R_{m n}$ (left) and $Z_{m n}$ (right). Two harmonics $\left(\tilde{n} \equiv n / N_{f p}, m\right)$ are shown. The top pair is for $(\tilde{n}, m)=(-3,1)$, to which $P_{1}$ is sensitive, and the bottom pair is for $(\tilde{n}, m)=(1,5)$, to which the kink is relatively sensitive. The size of the domain shown $\left(z_{j} \sim .02 \mathrm{~m}\right)$ is appreciable, large enough to encompass C82 as well as C10.

Probably because of the symmetrizing action of the optimizer in creating $\mathrm{C} 10$, most directions $Z_{j}$ resemble the top pair: the fractional variation $P / P_{0}$ of $P_{1}\left(Z_{j}\right)$ is much larger than that for $P_{5}$ (reflecting the near-optimal value of the unperturbed $P_{10}$ in the denominator), and $P_{1}$ is a parabolic curve, with vertex often near $z_{j}=0$. For all $Z_{j}$, the variation of both $P_{i}$ is smooth and rather unstructured, approximable by the quadratic expansion (1).

In Fig. 4 are shown histograms of the fractional sensitivity over the $(\tilde{n}, m)-$ plane (hence for all $Z_{j}$ ), for a fixed value $d Z$ of each $z_{j}$ comparable to the maximum shown in the plots of Fig. 3. As for the $\boldsymbol{\xi}^{i}$ discussed above, the dominant $(\tilde{n}, m)$ are similar for $i=1-4$, and different from those for $i=5$.

Also because of the action of the optimizer, the linear approximation Eq.(2) is valid only for very small displacements $\mathbf{z}$ (fractions of a millimeter) for some $Z_{j}$, much smaller than the values $(\sim 1 \mathrm{~cm})$ used to compute $\mathbf{G}$. Thus, the SVD displacements $\boldsymbol{\xi}^{i}$ shown earlier are not quantitatively correct.

However, as noted, the quadratic form (1) appears a good approximation for perturbations $\mathbf{z}$ on the order of a few centimeters. The simple $\mathbf{Z}$-space topography in the vicinity of $\mathrm{C} 10$ suggests that we can obtain a highly tractable model for study in this sizeable region. E.g., from Eq.(1) one can compute the control matrix for any $\mathbf{Z}$ in this region, via

$$
G_{i j}(\mathbf{Z}) \equiv \partial p_{i}(\mathbf{Z}) / \partial z_{j}=G_{i j}\left(\mathbf{Z}_{0}\right)+H_{i j k} z_{k},
$$

and from this, find the correct $\boldsymbol{\xi}^{i}$ at any $\mathbf{Z}$, along with the extrema of the $P_{i}$, etc.

Accordingly, we are in the process of computing both $G_{i j}$ and $H_{i j k}$ around the $\mathrm{C} 10$ design point. Since the number of required points $\mathbf{Z}$ to do this is large $(\simeq$ $2 N_{z}^{2}$ ), reducing the dimensionality of the $\mathbf{Z}$-space as much as possible is important, an issue addressed further below. 


\section{Other QAS Design Points}

C10 was arrived at along an involved path of human interaction with the optimizer, and it is unclear that other regions of $\mathbf{Z}$-space, which would have been reached from different starting points, might not yield superior configurations. Thus, we are starting to study other proposed QAS configurations $[4,5]$ with the same methods, and to consider the variation of the $P_{i}$ as one moves from one such point $\mathbf{Z}_{0}$ to another.

\subsection{The path from $\mathrm{C} 10$ to $\mathrm{C} 82$}

We begin by considering the $P_{i}$ along a straight-line trajectory $\mathbf{Z}=\mathbf{Z}_{C 10}+$ $\alpha\left(\mathbf{Z}_{C 82}-\mathbf{Z}_{C 10}\right)$ connecting $\mathrm{C} 10$ with $\mathrm{C} 82$, as $\alpha$ runs from 0 to 1 . These two configurations are fairly close in $\mathbf{Z}$-space. We may quantify this by introducing the simple norm: $|\mathbf{Z}| \equiv\left(\sum_{j} Z_{j}^{2}\right)^{1 / 2}$. With this definition, $\left|\mathbf{Z}_{C 82}-\mathbf{Z}_{C 10}\right| \simeq .041 \mathrm{~m}$, in comparison with the much larger 'distance' to PG1 (see below), $\left|\mathbf{Z}_{P G 1}-\mathbf{Z}_{C 10}\right| \simeq$ $.228 \mathrm{~m}$

C82 was obtained from C10 in an effort to stabilize the kink. The level of QAness was slightly degraded in compensation. This is borne out by the $P_{i}$ 's along the straight-line path in $\mathbf{Z}$-space, shown in Fig. 5. While the kink growth rate falls off to an acceptably low value $\left(\lambda_{C 82} / \lambda_{C 10} \simeq .05\right), P_{1}$ actually moves to a somewhat lower value (better quasisymmetry) about midway along the trajectory, and then rises at $\mathrm{C} 82$ to a value slightly larger than for $\mathrm{C} 10$.

\subsection{The path from $C 10$ to $P G 1$}

Configuration PG1 is characterized by[5] much better kink stability $(\lambda>0)$ than $\mathrm{C} 10$ or $\mathrm{C} 82$, but substantially worse quasisymmetry, mainly due to a large mirror field $B_{m=0, \tilde{n}=1}$ present to assure ballooning stability. As indicated above, its separation from $\mathrm{C} 10$ in $\mathbf{Z}$-space is far greater than that of $\mathrm{C} 82$, and is generally considered to be in a quite different region of $\mathbf{Z}$. Nevertheless, as one sees in the early results in Figs. 6 and 7, even over this relatively large distance the $P_{i}$ do not fluctuate wildly, but instead vary smoothly, and almost monotonically, in a manner consistent with the qualitative description of the physics differences given just above between the 2 stellarators.

Applying the same tools to PG1 as described above for C10, one finds sensitivity histograms for the $\boldsymbol{\xi}^{i}$ which resemble those for C10. Again, those for $i=1-4$ are similar to each other (and to those for C10), and differ from that for $i=5 . \xi^{5}$, which reduces the kink growth rate, is found to enhance the positive triangularity 
which PG1 possesses in the half-period $\tilde{\zeta}=\pi$, consistent with tokamak-based intuition on kink stabilization, an effect opposite that found for $\mathrm{C} 10$, which as noted earlier has negative triangularity at $\tilde{\zeta}=\pi$.

\section{Reducing the dimensionality of $\mathrm{Z}$ :}

Currently, the dimensionality $N_{z}$ of the search space and $N_{z}-M_{p}$ of its null subspace are large ( 78 and 73 , resp.). The smaller we can make these, the better, for the both numerical requirements and for fostering understanding. We expect to be able to reduce $N_{z}$ appreciably, by several means.

First, we expect to reduce $N_{z}$ by about a factor of 2 due to a redundancy in the present representation. In the calculations presented so far, each $R_{m n}$ and $Z_{m n}$ harmonic is independently varied. However, these variations are not, in fact, independent since the poloidal angle variable is not uniquely defined. In calculating the Control and Hessian matrices [Eq.(1)] one should use the $N_{z}^{*}<N_{z}$ linear combinations of the $R_{m n}, Z_{m n}$ that define normal displacements to the plasma boundary.

There are various ways of defining nonredundant normal perturbations to the plasma boundary. One way is to use the 'quasipolar' representation of Hirshman and Breslau[6] which is adopted by the VMEC optimization code. For the C10 configuration, this leads to a reduction in the number of independent variables from $N_{z}=78$ to $N_{z}^{*}=32$.

Such redundancy in the representation contributes extra nonphysical dimensions to the null space. Additionally, we should be able to further reduce $N_{z}^{*}$ by making use of our understanding of the topography of $\mathbf{Z}$-space. For example, the sensitivity diagrams illustrated by Fig. 4, along with the knowledge that the $P_{i}(\mathbf{Z})$ vary only slowly, may allow us to get adequate physics performance from using only a subset of the $Z_{i}$ presently kept.

\section{Summary}

We have now set up most of the machinery needed to do the control-matrix analysis of NCSX, and have presented some initial findings here, mostly for the C10-C82 family of stellarators our optimizer has led the NCSX group to. For the 1 st time, we are getting a picture of the topography of the configuration space $\mathbf{Z}$ in which the optimizer has been searching for good stellarators. The controlmatrix method would be applicable and useful even in a $\mathbf{Z}$-space which was highly involuted, but instead it appears that the space is rather smooth and unstructured. In an appreciable neighborhood of $\mathrm{C} 10$, it appears the $P_{i}$ may be modeled by a 
quadratic function of $\mathbf{z}=\mathbf{Z}-\mathbf{Z}_{0}$, and vary with little structure even over a scale comparable to the distance from $\mathrm{C} 10$ to PG1. We now plan to use this knowledge to provide a quantitatively correct control matrix $\mathbf{G}(\mathbf{Z})$ and $\boldsymbol{\xi}^{i}$. That the quadratic approximation (1) is valid should considerably facilitate our efforts to obtain an understanding of where the optima lie, and why. We have also begun studying other base configurations such as PG1, to be able to see which characteristics are generic and which reflect important differences in stellarator concepts.

The 4 different transport figures of merit $(i=1-4)$ produce boundary displacements $\boldsymbol{\xi}^{i}(\theta, \zeta)$ similar in appearance, and these differ from that for the kink $(i=5)$. However, the $\mathbf{G}$-matrix eigenvalues $w_{i}$ show that $\boldsymbol{\xi}^{i=1,5}$ are linearly independent and not nearly collinear. Correspondingly, for both C10 and PG1, the sensitivity histograms for $i=1-4$ also resemble one another, and differ from that for $i=5$.

For $\mathrm{C} 10, \boldsymbol{\xi}^{5}$ provides the outboard indentation previously observed to stabilize the kink, enhancing C10's negative triangularity at $N_{f p} \zeta=\pi$, while for PG1, $\xi^{5}$ enhances its positive triangularity, consistent with tokamak intuition on kink stabilization.

The dimensionality $N_{z}$ of the search space and $N_{z}-M_{p}$ of its null subspace have been large. We expect to be able to considerably reduce these by removing the redundancy which exists in the current $\left(R_{m n}, Z_{m n}\right)$ boundary representation, and by making use of our enhanced understanding of the topographic features (scales of variation and sensitivities) of the $P_{i}(\mathbf{Z})$. From this reduction we should be able to express the physics characteristics of these stellarators in terms of a relatively modest set of parameters, which should aid in both our understanding, and in focussing the optimizer.

Finally, the work reported here takes as its free 'control knobs' $Z_{j}$ displacements of the plasma boundary. However, exactly the same method may be used to study how a given set of coil currents (with perturbations $\delta \mathbf{I}$ ) could produce a range of physics behavior $\mathbf{p}$ for experimental flexibility. One still has relation (2) between the boundary shape and the physics parameters, and using free-boundary equilibrium runs, one may additionally relate the $\delta \mathbf{I}$ to $\mathbf{z}$ :

$$
\mathbf{z}=\mathbf{C} \cdot \delta \mathbf{I}
$$

Combining these, one has

$$
\mathbf{p}=\mathbf{G}_{2} \cdot \delta \mathbf{I},
$$

with $\mathbf{G}_{2} \equiv \mathbf{G} \cdot \mathbf{C}$, of the same form as Eq.(2), and so amenable to the same SVDbased analysis. 


\section{References}

[1] A. Reiman, G. Fu, S. Hirshman, D. Monticello, H. Mynick, et al., European Physical Society Meeting on Controlled Fusion and Plasma Physics Research, Maastricht, the Netherlands, June 14-18, 1999, (European Physical Society, Petit-Lancy, 1999).

[2] M.Yu. Isaev, M.I. Mikhailov, D.A. Monticello, H.E. Mynick, A.A. Subbotin, L.P. Ku, A.H. Reiman, Phys. Plasmas 63174 (1999).

[3] Wm. H. Press, et al., Numerical Recipes in Fortran 77, (Cambridge University Press, 1996), p.51ff.

[4] N. Nakajima, M. Yokoyama, M. Okamoto, J. Nührenberg, Plasma Physics Reports 23, 460 (1997).

[5] P. Garabedian, L.P. Ku, Phys. Plasmas 6, 645 (1999).

[6] S.P. Hirshman and J. Breslau, Phys. Plasmas 5, 2664 (1998). 


\section{Figures}

Fig. 1. Contour and surface plots of $\xi^{1}$ and $\xi^{5}$ for $\mathrm{C} 10$, showing the character of the perturbation which changes ripple parameter $P_{1}=\chi_{1}^{2}$ without modifying kink stability $(i=1)$ and that which changes the kink growth rate $P_{5}=\lambda$ without modifying the ripple $(i=5)$.

Fig. 2. Poloidal cross-sections of unperturbed (solid curve) and perturbed boundaries of $\mathrm{C} 10$, at $\tilde{\zeta}=0$ (top) and $\pi$ (bottom).

Fig. 3. Variation of $P_{1}=\chi_{1}^{2}$ and $P_{5}=\lambda$ with deformation amplitude (in meters), for changes of the given $R_{m n}$ (left) and $Z_{m n}$ (right), and for $(\tilde{n}, m)=(-3,1)$ (top) and (1,5) (bottom).

Fig. 4. Histograms of the fractional variation $P_{i} / P_{i 0}$ over the full $(\tilde{n}, m)-$ plane for $\mathrm{C} 10$, for variations $d R_{m n}=.025 \mathrm{~m}$ (left), and $d Z_{m n}=.04 \mathrm{~m}$ (right), and for $P_{1}$ (top) and $P_{5}$ (bottom).

Fig. 5. Plot of the fractional variation $P_{i} / P_{i 0}$ for $i=1-5$ along a straight-line path in $\mathbf{Z}$-space from $\mathrm{C} 10(\alpha=0)$ to $\mathbf{C} 82(\alpha=1)$. These have a $\mathbf{Z}$-space distance between them of $.041 \mathrm{~m}$.

Fig. 6. Plot of the fractional variation $P_{i} / P_{i 0}$ for $i=1-5$ along a straight-line path in Z-space from $\mathrm{C} 10(\alpha=0)$ to PG1 $(\alpha=1)$. These have a $\mathbf{Z}$-space distance between them of $.228 \mathrm{~m}$.

Fig. 7. Blowup of Fig. 6, to show more clearly the variation in $P_{5}$. 

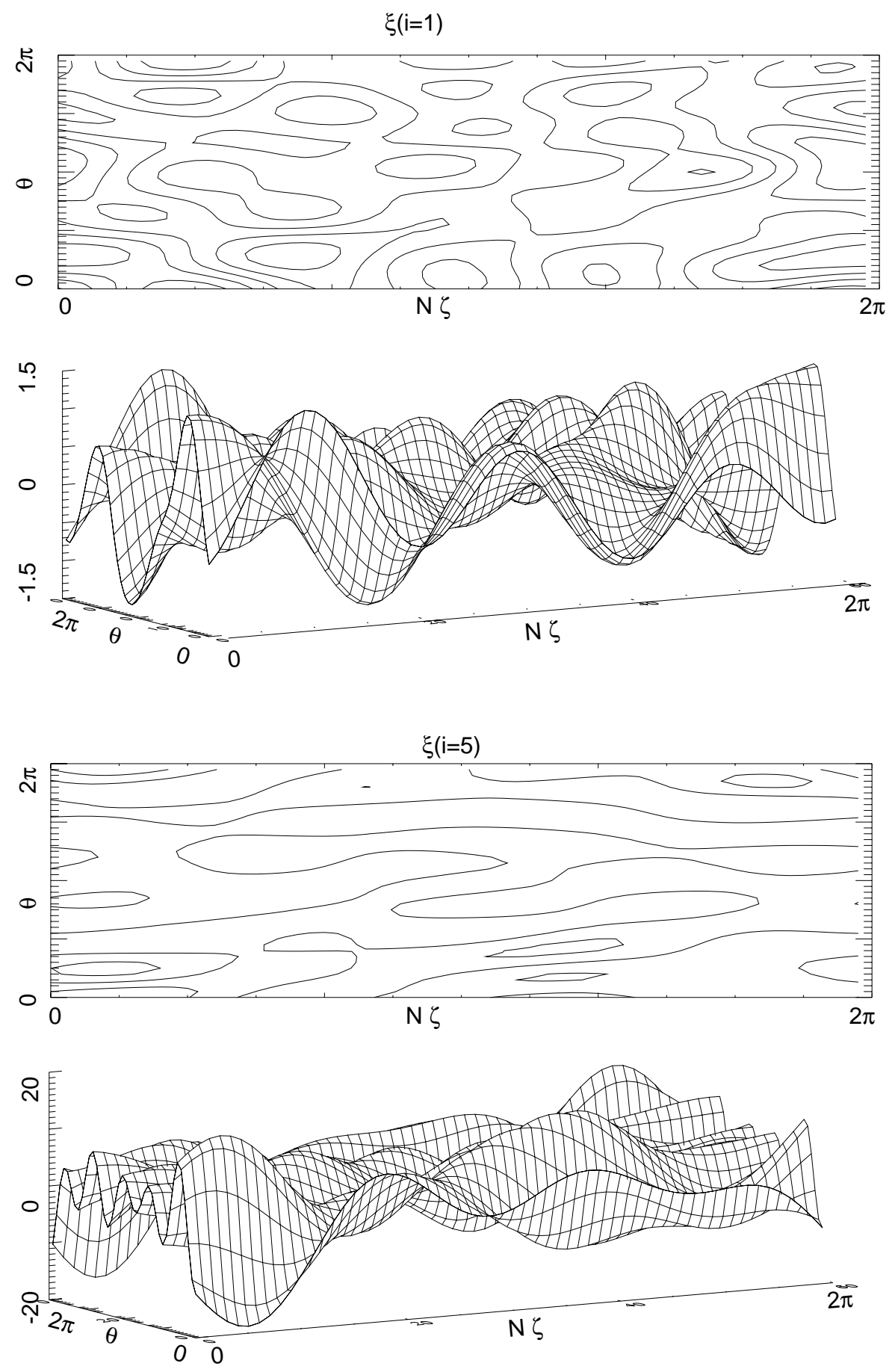

Figure 1: 

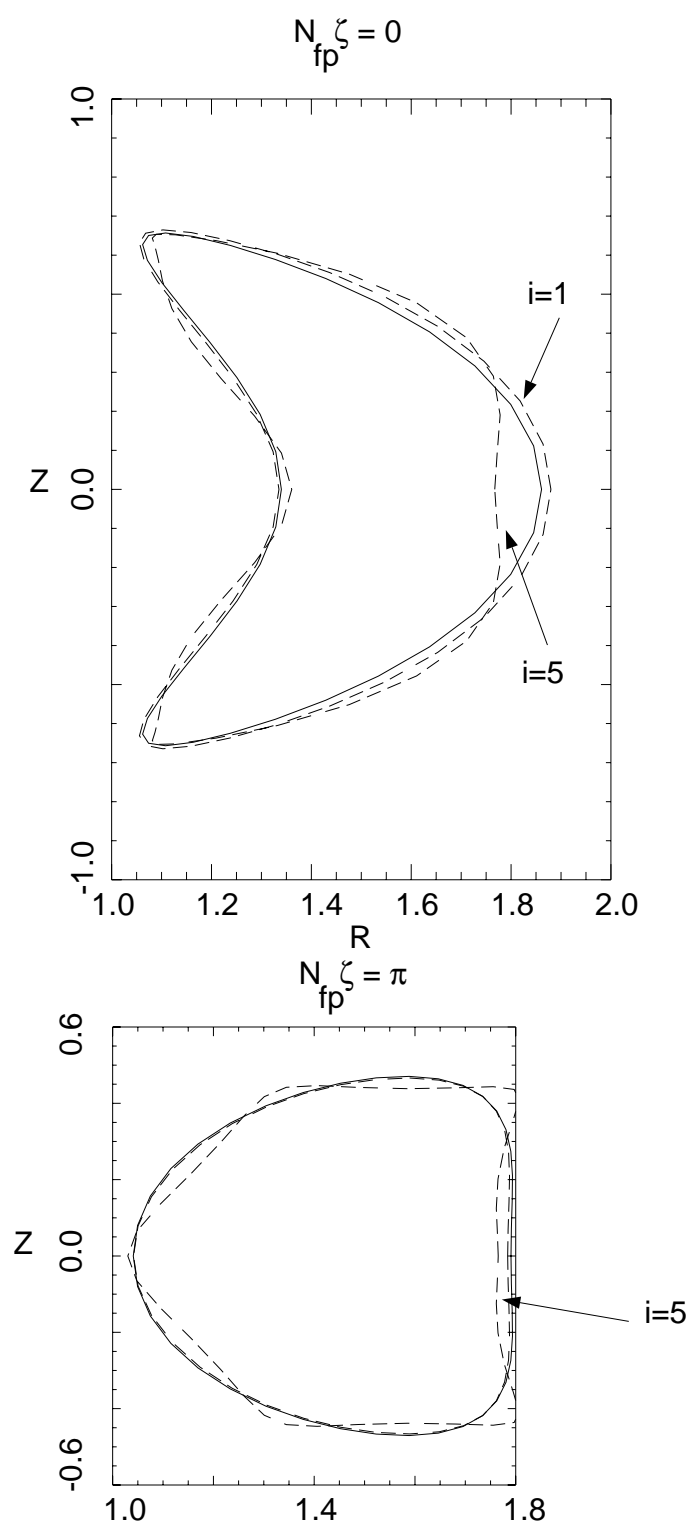

Figure 2: 

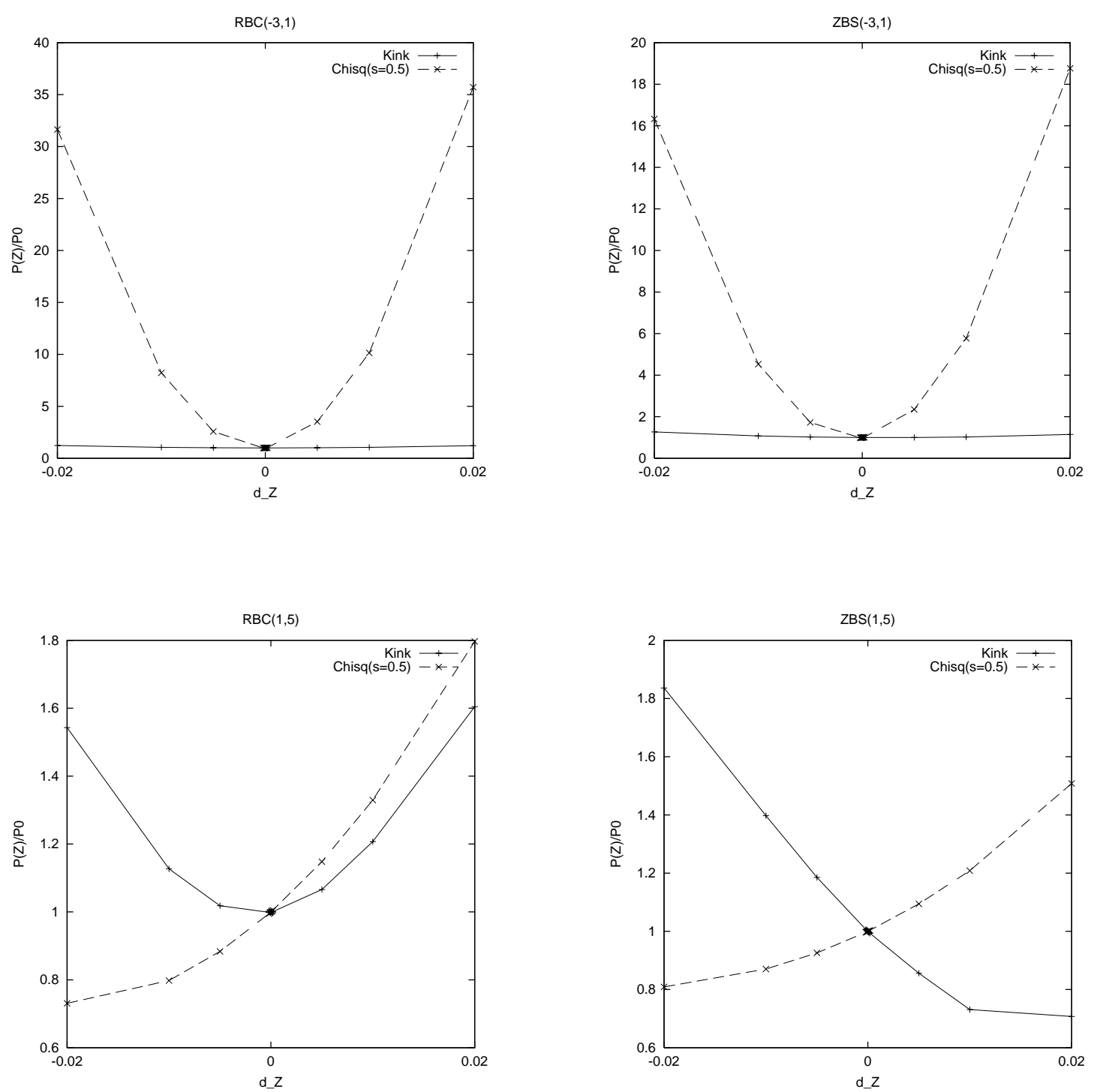

Figure 3: 


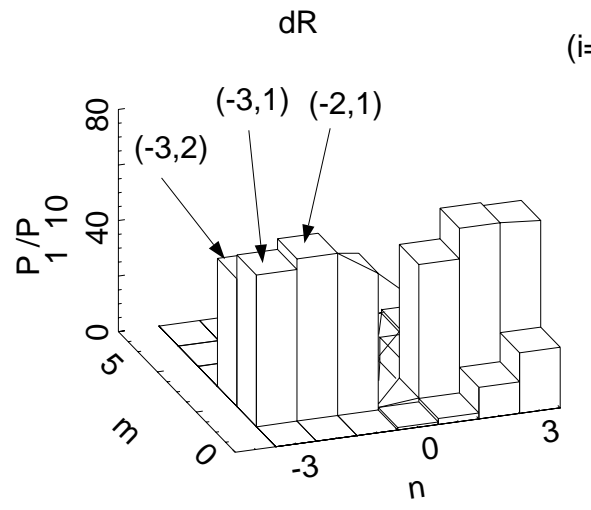

(i=1)

$d Z$
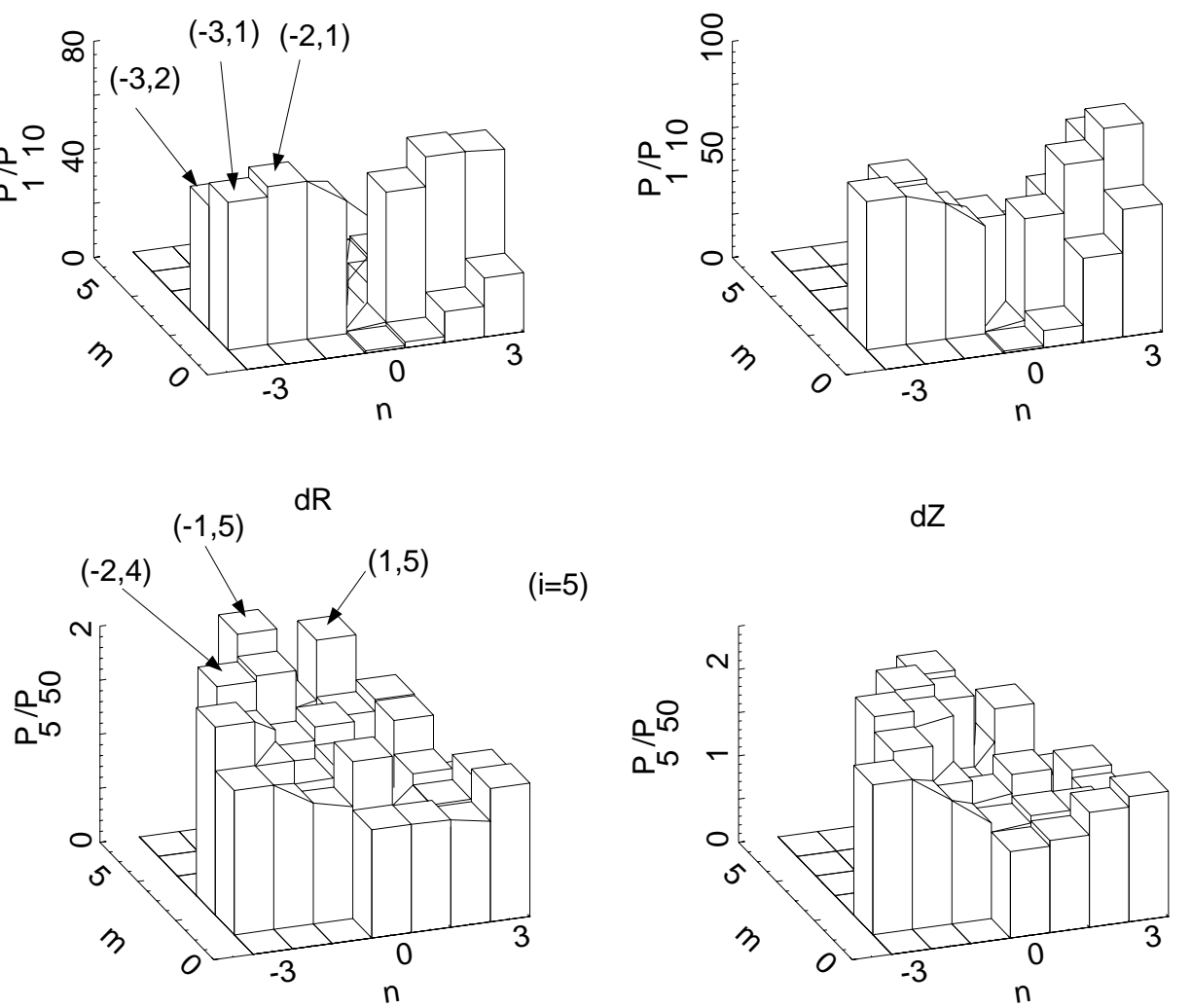

Figure 4: 


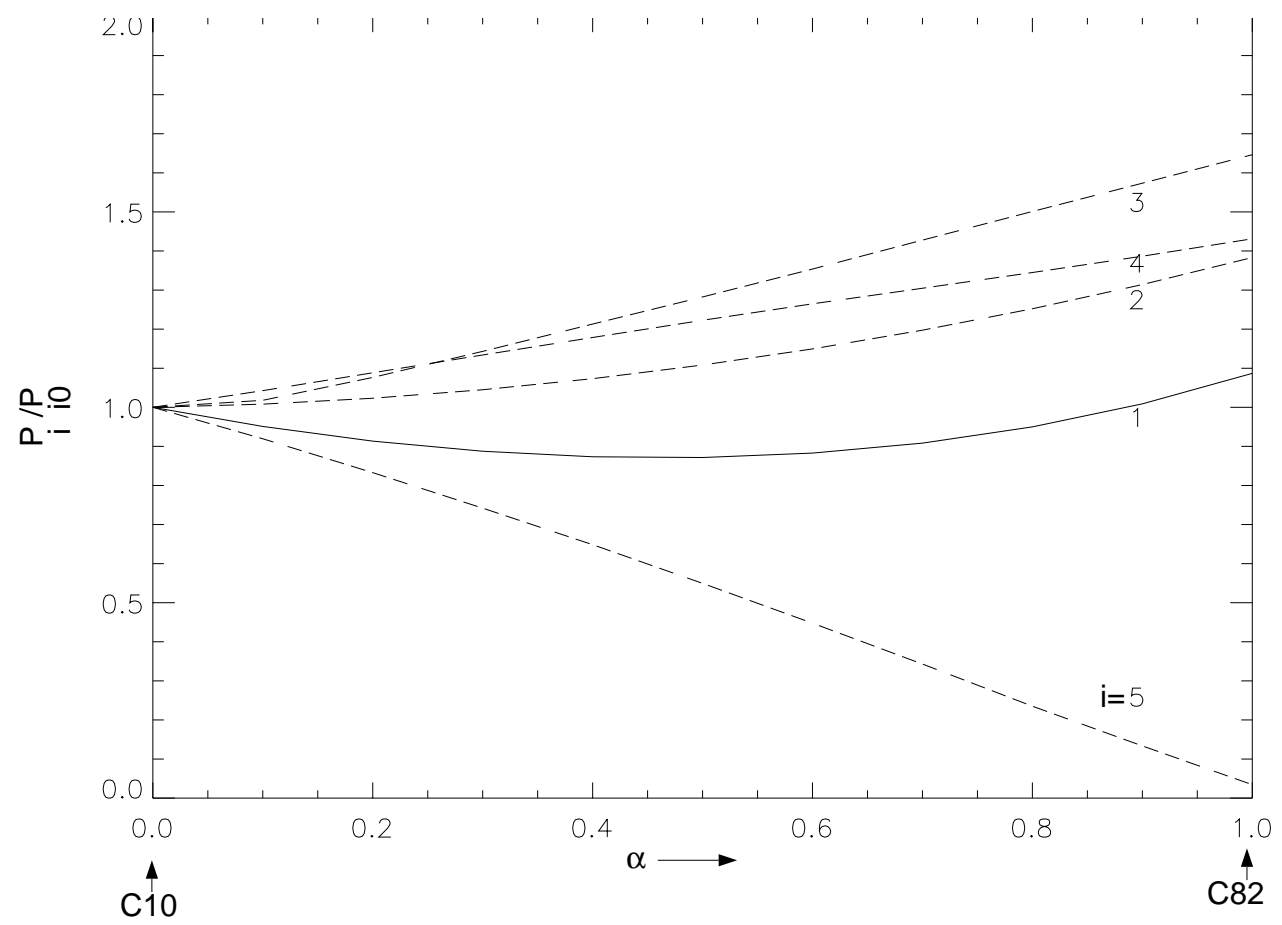

Figure 5: 


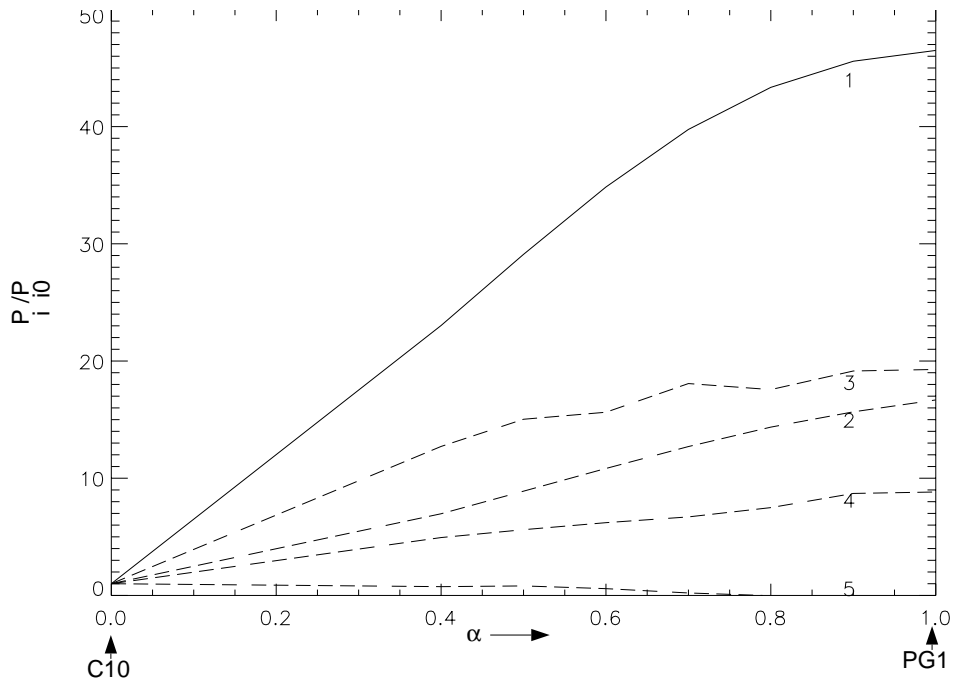

Figure 6:

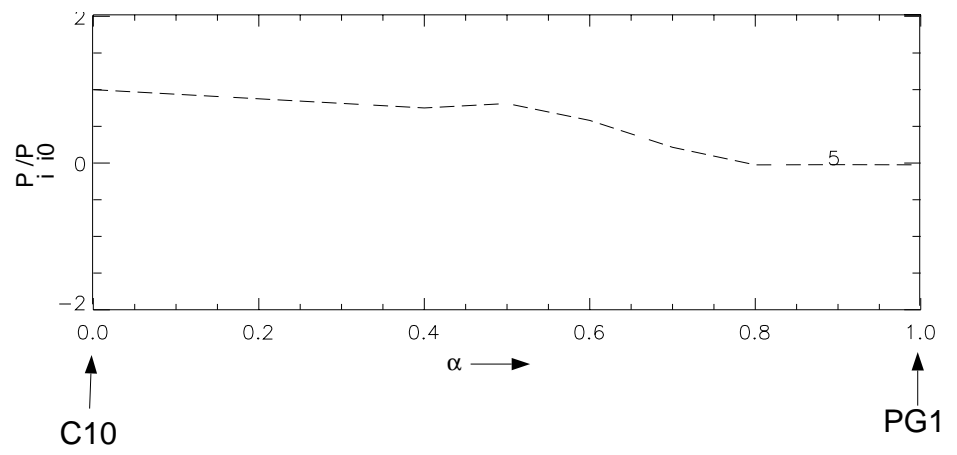

Figure 7: 